\title{
Quantum transport of a two-dimensional electron gas in a spatially modulated magnetic field
}

\author{
F. M. Peeters \\ Department of Physics, University of Antwerp (UIA), B-2610 Antwerpen, Belgium \\ P. Vasilopoulos \\ Department of Physics, Concordia University, Montréal, Canada H3G 1 M8
}

(Received 10 August 1992)

\begin{abstract}
Electrical transport properties of a two-dimensional electron gas are studied in the presence of a perpendicular magnetic field $B$ modulated weakly and periodically along one direction, $\mathbf{B}=$ $\left(B+B_{0} \cos K x\right) \hat{\mathbf{z}}$, with $B_{0} \ll B, K=2 \pi / a$, and $a$ being the period of the modulation. $B_{0}$ is taken constant or proportional to $B$. The Landau levels broaden into bands and their width, proportional to the modulation strength $B_{0}$, oscillates with $B$ and gives rise to oscillations in the magnetoresistance at low $B$. These oscillations reflect the commensurability between the cyclotron diameter at the Fermi level and the period $a$ and consequently they are distinctly different from the Shubnikov-de Haas ones, at higher $B$, in period and temperature dependence. The bandwidth at the Fermi energy can be one order of magnitude larger, at low $B$, than that of the electric case for equal modulation strengths. The resulting magnetoresistance oscillations have a much higher amplitude than those of the electric case with which they are out of phase. Explicit asymptotic expressions are derived for the temperature dependence of the transport coefficients. The case when both electric and magnetic modulations are present is also considered. The position of the resulting oscillations depends on the ratio $\delta$ between the two modulation strengths. When the modulations are out of phase there is no shift in the position of the oscillations when $\delta$ varies and for a particular value of $\delta$ the oscillations are suppressed.
\end{abstract}

\section{INTRODUCTION}

The magnetoresistance oscillations of a two-dimensional electron gas (2DEG) subject to periodic electric (or potential) weak modulations, along one or two directions, also called Weiss oscillations, are now well established both experimentally, ${ }^{1}$ and theoretically. ${ }^{2,3}$ The situation is mostly clear in the case of one-dimensional (1D) modulations where the oscillations reflect the commensurability between two length scales: the cyclotron diameter at the Fermi level, $2 R_{c}=2 \sqrt{2 \pi n_{e}} \ell^{2}$ (where $n_{e}$ is the electron density and $\ell=\sqrt{\hbar / e B}$ the magnetic length) and the period $a$ of the modulation.

In this paper we consider electrical magnetotransport of a $2 \mathrm{DEG}$ in the presence of a weak $1 \mathrm{D}$ periodic modulation (of strength $B_{0}$ ) of the magnetic field. A partial and brief account of transport, for $B_{0}$ constant, has been reported in Refs. 4 and 5 while Ref. 6 treated the elementary excitations of such a system. Experimentally this magnetic modulation of the 2DEG could be achieved by covering the heterostructure with stripes of magnetic materials. If the latter are replaced by superconducting materials the modulation is strong. The feasibility of the latter technique was demonstrated recently by Heym et $a .^{7}$ who were able to cover a heterostructure with a type-II superconducting film. Here we treat only the weak modulation and take $B_{0}$ to be constant or proportional to $B$. The first choice is made for a clearer comparison of the results with those of the electric modulation of constant strength but the second one is expected to be more realistic for both methods of magnetic modulation mentioned above. Moreover, we present asymptotic expressions for the conductivity tensor, missing from Refs. 4 and 5, that show explicitly both the Weiss and Shubnikov-de Haas (SdH) oscillations. In addition, we consider the case where both electric and magnetic modulations are present. Experimentally from the above-mentioned methods of magnetic modulation one expects that the magnetic or superconducting stripes act like electrical gates and induce an electric modulation of the 2DEG. To our knowledge this case has not been treated. A corresponding nontrivial result is that the Weiss oscillations are suppressed when the modulations are out of phase and the ratio of their strengths has a particular value.

In the next section we present the energy spectrum corresponding to the one-electron Hamiltonian, when only the magnetic modulation is present, and discuss some of its consequences. In Sec. III we give the results for the magnetoresistance tensor and in Sec. IV those for the case when both electric and magnetic modulations are present. Concluding remarks follow in the last section.

\section{ENERGY SPECTRUM}

We consider a $2 \mathrm{DEG}$, in the $(x, y)$ plane, subject to the magnetic field $\mathbf{B}=\left(B+B_{0} \cos K x\right) \hat{\mathbf{z}}$, where $K=2 \pi / a$, 
and $a$ is the modulation period. Here we will consider only the case of a weak modulation, i.e., $B_{0} \ll B$; this will allow us to make a direct analogy with the case of weak electric modulation and thus exploit the previous experience. ${ }^{1-4}$ In the Landau gauge the vector potential $\mathbf{A}$ that describes this periodic magnetic field is $\mathbf{A}=\left[0, B x+\left(B_{0} / K\right) \sin (K x), 0\right]$ with $B_{0} \ll B$. In the effective-mass $\left(m^{*}\right)$ approximation the corresponding one-electron Hamiltonian is given by $H=(\mathbf{p}+e \mathbf{A}) / 2 m^{*}$ and can be written as

$$
\begin{aligned}
H= & {\left[p_{x}^{2}+\left(p_{y}+e B x\right)^{2}\right] / 2 m^{*} } \\
& +\left(\omega_{0} / K\right)\left(p_{y}+e B x\right) \sin K x \\
& +\left(m^{*} \omega_{0}^{2} / 4 K^{2}\right)(1-\cos 2 K x)
\end{aligned}
$$

here $p_{\mu}(\mu=x, y)$ is the momentum operator, $\omega_{0}=$ $e B_{0} / m^{*} \ll \omega_{c}=e B / m^{*}$, and $\omega_{c}$ is the cyclotron frequency. The first line on the right-hand side (RHS) of Eq. (1) is the Hamiltonian of a free electron in a magnetic field. The corresponding normalized eigenvectors are $\psi_{n k_{y}}=e^{i k_{y} y} \phi_{n}\left(x+x_{0}\right) / \sqrt{L_{y}}$, where $x_{0}=-\ell^{2} k_{y}=$ $-\hbar k_{y} / e B$ is the center coordinate of the cyclotron orbit, $L_{y}$ is the width of the sample in the $y$ direction, and where $n$ is the Landau-level index. $\phi_{n}(x)$ is the wellknown harmonic-oscillator wave function and the corresponding eigenvalues are $E_{n k_{y}}=(n+1 / 2) \hbar \omega_{c}$ and are degenerate with respect to the wave vector $k_{y}$ along the $y$ direction.

Because of the inequality $B_{0} \ll B$ we can consider the last two terms on the RHS of Eq. (1) as perturbation and evaluate the energy correction to $E_{n k_{y}}$ given above by first-order perturbation theory using the unperturbed eigenstates $\psi_{n k_{y}}$. The result is

$$
\begin{aligned}
E_{n k_{y}}= & (n+1 / 2) \hbar \omega_{c}+\hbar \omega_{0} G_{n}(u) \cos K x_{0} \\
& +\left(m^{*} \omega_{0}^{2} / 4 K^{2}\right)\left[1-e^{-2 u} L_{n}(4 u) \cos 2 K x_{0}\right]
\end{aligned}
$$

where $u=K^{2} \ell^{2} / 2, L_{n}(z)$ is a Laguerre polynomial, and

$$
G_{n}(u)=e^{-u / 2}\left[L_{n}(u) / 2+L_{n-1}^{1}(u)\right]
$$

Since $x_{0}=-\ell^{2} k_{y}$, the $k_{y}$ degeneracy of the energy levels is lifted and the energy levels broaden into bands as in the case of a $1 D$ potential modulation. In the following we will retain only terms of order $\omega_{0}$ in the energy spectrum and neglect terms of higher order. The correspondence with the electric case ${ }^{3}$ for a modulation strength $V_{0}$ is as follows: $G_{n}(u)=-\partial F_{n}(u) / \partial u \rightarrow F_{n}(u)=$ $\exp (-u / 2) L_{n}(u)$. In a typical experiment the magnetic field $B$ is weak and many Landau levels are occupied. It then turns out that it is a good approximation to take the large $n$ limit of the Laguerre polynomials that appear in $G_{n}(u)$ and $F_{n}(u)$. For the electric modulation the width of the Landau level at the Fermi energy is given by ${ }^{3}$

$$
2 V_{0}\left|F_{n}(u)\right| \approx 2 V_{0}\left(2 / \pi K R_{c}\right)^{1 / 2}\left|\cos \left(K R_{c}-\pi / 4\right)\right|
$$

whereas now $V_{0}$ is replaced by $\hbar \omega_{0}$ and $F_{n}(u)$ by $G_{n}(u)$. The resulting bandwidth for large $n$ is

$$
\begin{aligned}
2 \hbar \omega_{0}\left|G_{n}(u)\right| \approx & 2 \hbar \omega_{0}\left(a k_{F} / 2 \pi\right)\left(2 / \pi K R_{c}\right)^{1 / 2} \\
& \times\left|\sin \left(K R_{c}-\pi / 4\right)\right|
\end{aligned}
$$

here $R_{c}=\left(n_{F}+1 / 2\right)^{1 / 2} \ell$ is the cyclotron orbit at the Fermi energy and $n_{F}$ is defined as the largest integer contained in $\left(E_{F}-1 / 2\right) / \hbar \omega_{c}$. From Eq. (4b) we obtain the flat-band condition as $2 R_{c} / a=i+\frac{1}{4}$; the maximum bandwidth occurs for $2 R_{c} / a=i+\frac{3}{4}$, with $i=0,1,2, \ldots$.

Equations (4a) and (4b) were obtained using the asymptotic expressions for the Laguerre polynomials and are valid in the low magnetic-field limit, typically $B \leq 1$ $\mathrm{T}$, in which case $n \gg 1$. Comparing Eq. (4a) with Eq. (4b) we see that (1) the bandwidths are out of phase, and (2) the amplitude in the magnetic modulation case is larger by a factor $a k_{F} / 2 \pi=\sqrt{n_{e} a^{2} / 2 \pi}$ (8.6 for $n_{e}=3 \times 10^{11} / \mathrm{cm}^{2}$ and $a=3820 \AA$ ) than the corresponding amplitude in the electric case for equal modulation strengths. This is illustrated in Fig. 1 for the width of the Landau level at the Fermi energy. For the magnetic modulation we show the result $2\left|G_{n}(u)\right|$ (solid curve) as well as the asymptotic expression Eq. (4b) (dotted curve). The jumps in the solid curve are due to the fact that the integer $n_{F}$ changes discontinuously with $B$. The bandwidth for the electric modulation, when $V_{0}=\hbar \omega_{0}$, is shown as the short-dashed curve.

It is instructive to give a classical derivation of the energy correction due to the modulation since the magnetic field is weak and the quantum number $n$ large. In classical mechanics the electron motion along the $x$ and $y$ directions is described by $x(t)=x_{0}+R_{c} \sin \left(\omega_{c} t+\varphi\right)$ and

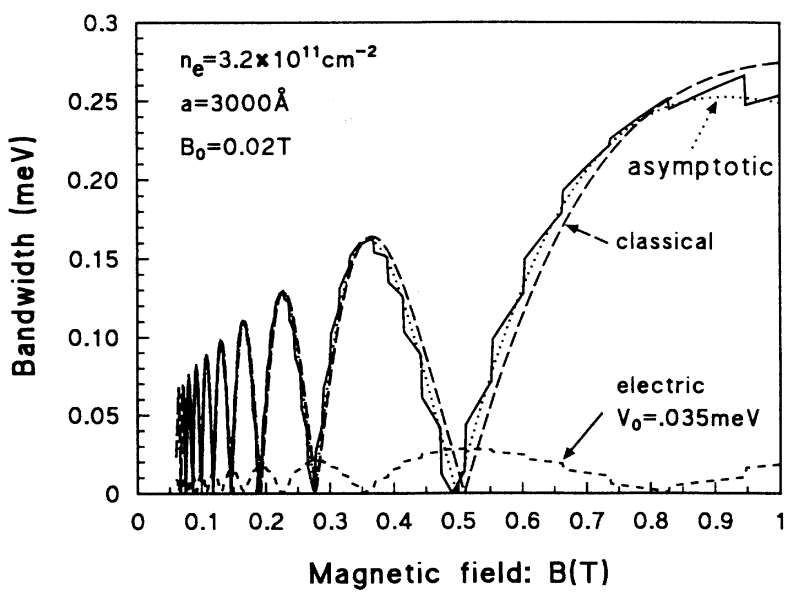

FIG. 1. The width of the Landau level at the Fermi energy as a function of the magnetic field. The solid curve is the exact numerical result for a constant modulation of strength $B_{0}=0.02 \mathrm{~T}$. The dotted and long-dashed curves are the corresponding asymptotic and classical results. The shortdashed curve is the result for an electric modulation of equal strength, $V_{0}=\hbar \omega_{0}$. 
$y(t)=y_{0}+R_{c} \cos \left(\omega_{c} t+\varphi\right)$, respectively, where $x_{0}, y_{0}$ are the center coordinates and $\varphi$ is a phase factor. Without loss of generality, $\varphi$ can be taken equal to zero. The contribution to the potential energy $U$ due to the mod- ulation of the magnetic field is $U=-e \mathbf{A}_{0} \cdot \mathbf{v}$ where $\mathbf{v}$ is the electron velocity and $\mathbf{A}_{0}=\left(e B_{0} / K\right) \sin (K x) \hat{\mathbf{y}}$. The mean value $\langle U\rangle$ over the period $t_{0}$ of the cyclotron motion is

$$
\begin{aligned}
\langle U\rangle=\frac{1}{t_{0}} \int_{0}^{t_{0}} U(t) d t & =e B_{0} \omega_{c}\left(R_{c} / K\right) \cos K x_{0} \frac{1}{t_{0}} \int_{0}^{t_{0}} \sin \omega_{c} t \sin \left(K R_{c} \sin \omega_{c} t\right) d t \\
& =\hbar \omega_{0}\left(R_{c} / K \ell^{2}\right) J_{1}\left(K R_{c}\right) \cos K x_{0}
\end{aligned}
$$

where $J_{1}(z)$ is the Bessel function of order 1. In the limit $K R_{c} \gg 1$, i.e., for weak magnetic fields, $\langle U\rangle$ becomes equal to the second term of Eq. (2) thus resulting in the bandwidth given by Eq. (4b). The bandwidth resulting from Eq. (5), prior to taking the limit $K R_{c} \gg 1$, is shown in Fig. 1 by the long-dashed curve and is indicated as "classical."

In the absence of modulation we have $v_{x}=v_{y}=0$ for all eigenstates. Because the modulation lifts the $k_{y}$ degeneracy we find to order $\hbar \omega_{0}$,

$$
v_{y}=\frac{1}{\hbar} \frac{\partial E_{n, k_{y}}}{\partial k_{y}}=-\frac{2 \omega_{0}}{K} u G_{n}(u) \sin K x_{0}
$$

while $v_{x}=0$. The fact that $v_{y}$ is no longer zero has important consequences for transport which will be detailed in the next section.

\section{THE RESISTIVITY TENSOR}

\section{A. Conductivities}

For weak electrical fields, i.e., for linear responses, and weak scattering the conductivity tensor $\sigma_{\mu \nu}$ has been evaluated, for the case of electric modulations, in Ref. 3. In general, we have $\sigma_{\mu \nu}^{\mathrm{I}}(\omega)+\sigma_{\mu \nu}^{\mathrm{II}}(\omega)$, where $\omega$ is the frequency. The relevant quantity $\sigma_{\mu \nu}^{\mathrm{I}}(0) \equiv \sigma_{\mu \nu}$ for dc transport is, in general, the sum of a diffusive (dif) and of a collisional (col) contribution given by ${ }^{3}$

$$
\sigma_{\mu \nu}^{\mathrm{dif}}=\frac{\beta e^{2}}{\Omega} \sum_{\zeta} f_{\zeta}\left(1-f_{\zeta}\right) \tau\left(E_{\zeta}\right) v_{\mu}^{\zeta} v_{\nu}^{\zeta}
$$

and

$$
\sigma_{\mu \mu}^{\mathrm{col}}=\frac{e^{2}}{2 \Omega} \sum_{\zeta \zeta^{\prime}} f_{\zeta}\left(1-f_{\zeta^{\prime}}\right) W_{\zeta \zeta^{\prime}}\left(\alpha_{\mu}^{\zeta}-\alpha_{\mu}^{\zeta^{\prime}}\right)^{2}
$$

respectively. Here, $W_{\zeta \zeta^{\prime}}$ is the transition rate between the unperturbed one-electron states $|\zeta\rangle$ and $\left|\zeta^{\prime}\right\rangle(|\zeta\rangle \equiv$ $\psi_{n k_{y}}$ ) given by the golden rule, $E_{\zeta}$ is the corresponding eigenvalue given by Eq. (2), $\tau\left(E_{\zeta}\right)$ is the relaxation time, and $f_{\zeta}$ the Fermi-Dirac function. Further, $\alpha_{\mu}^{\zeta}=\left\langle\zeta\left|r_{\mu}\right|\right.$ $\zeta\rangle$ is the mean value of the $\mu$ component of the position operator $r$ in the state $|\zeta\rangle$ and $v_{\mu}^{\zeta}$ the corresponding velocity: $v_{\mu}^{\zeta}=\left\langle\zeta\left|v_{\mu}\right| \zeta\right\rangle$. If $\mu \neq \nu$ the collisional contribution vanishes identically.

The dc component $\sigma_{\mu \nu}^{\mathrm{II}}$ is given ${ }^{3}$ by

$$
\begin{aligned}
\sigma_{\mu \nu}^{\mathrm{II}}=i \hbar \frac{e^{2}}{\Omega} \sum_{\zeta \neq \zeta^{\prime}} & f_{\zeta}\left(1-f_{\zeta^{\prime}}\right)\left\langle\zeta\left|v_{\mu}\right| \zeta^{\prime}\right\rangle \\
& \times\left\langle\zeta^{\prime}\left|v_{\nu}\right| \zeta\right\rangle \frac{1-e^{\beta\left(E_{\zeta}-E_{\zeta^{\prime}}\right)}}{\left(E_{\zeta}-E_{\zeta^{\prime}}\right)^{2}}
\end{aligned}
$$

and is independent of scattering in the limits taken.

A detailed evaluation of formulas (7)-(9) has been carried out in Ref. 3(b) for scattering by randomly distributed impurities of density $N_{I}$ and of interaction strength, in Fourier space, $U_{q}=\left(2 \pi e^{2} / \epsilon\right) / \sqrt{q^{2}+k_{s}^{2}} \approx$ $\left(2 \pi e^{2} / \epsilon\right) / k_{s}=U_{0}$ in the limit $q \ll k_{s} ;$ here $k_{s}$ is the inverse screening length and $\epsilon$ the dielectric constant. Then $1 / \tau\left(E_{\zeta}\right)=\sum_{\zeta^{\prime}} W_{\zeta \zeta^{\prime}}$ gives approximately $\tau\left(E_{\zeta}\right) \approx\left(\pi \ell^{2} \hbar^{2} / N_{I} U_{0}^{2}\right)^{1 / 2}$

In the absence of modulation, we have $v_{x}=v_{y}=0$ and the diffusion contribution vanishes. In its presence, we have $v_{x}=0$ but $v_{y} \neq 0$, cf. Eq. (6). Thus $\sigma_{x x}^{\text {dif }}(0)=0$, whereas $\sigma_{y y}^{\text {dif }}(0) \equiv \sigma_{y y}^{\text {dif }} \neq 0$. To order $B_{0}^{2}$ we find

$$
\sigma_{y y}^{\mathrm{dif}}=\frac{e^{2}}{h} \frac{2 \pi^{2} \tau}{\hbar} \frac{\ell^{2}}{a^{2}} \sum_{n}\left[\hbar \omega_{0} G_{n}(u)\right]^{2}\left(-\frac{\partial f(E)}{\partial E}\right)_{E=E_{n}}
$$

where $E_{n}=(n+1 / 2) \hbar \omega_{c}$. Comparing this result with that for electric modulation of strength $V_{0}$, Eq. (13) of Ref. 3(b), we see that it has the same structure: $V_{0} F_{n}(u)$ is replaced by $\hbar \omega_{0} G_{n}(u)$. In either case this contribution is proportional to the square of the bandwidth.

To evaluate the collisional contribution to order $B_{0}^{2}$ we must take into account the correction to the unperturbed eigenstate $\left|n k_{y}\right\rangle^{0}$ due to the weak 1D modulation. Using first-order perturbation theory we find the perturbed eigenstate $\left|n k_{y}\right\rangle$ as

$$
\left|n k_{y}\right\rangle=\left|n k_{y}\right\rangle^{0}+\sum_{n^{\prime} \neq n} \frac{V_{n n^{\prime}}}{E_{n}-E_{n^{\prime}}}\left|n^{\prime} k_{y}\right\rangle^{0}
$$

and from the sum over $n^{\prime}$ we consider only the nearest Landau levels $n^{\prime}=n \pm 1$ which give the dominant contribution. We obtain 


$$
V_{n, n+1}=\frac{\hbar \omega_{0}}{2 K \ell u} \sqrt{2(n+1)} e^{-u / 2}\left[(1-u) L_{n}(u)-(1+u) L_{n+1}(u)\right] \sin K x_{0}
$$

for $V_{n, n-1}$ we obtain the same expression multiplied first by -1 and followed by the change $n+1 \rightarrow n-1$. The rest of the relevant formulas and approximations as well as the procedure are similar to those of Ref. 3(b). The result is

$$
\sigma_{x x}^{\mathrm{col}} \approx \frac{e^{2}}{h} \frac{N_{I} U_{0}^{2}}{\pi \Gamma a} \sum_{n}\left\{(2 n+1) I_{n}+\Delta_{n}\right\}
$$

with

$$
\Delta_{n}=\frac{\omega_{0}^{2}}{\omega_{c}^{2} u^{2} K \ell} e^{-u}\left[n^{2} F_{-}^{2}+(n+1)^{2} F_{+}^{2}-n(n+1) F_{-} F_{+}\right] J_{n}
$$

$I_{n}$ and $J_{n}$ are given by Eqs. (24) and (27) of Ref. 3(b), respectively, and

$$
F_{ \pm}=(1 \pm u) L_{n \pm 1}(u)-(1 \mp u) L_{n}(u)
$$

Equation (13) has the same structure as Eq. (25) of Ref. 3(b) for the electric modulation: the prefactor and the first term in $\{\cdots\}$ are the same and the essential change in $\Delta_{n}$ is that in its prefactor $V_{0} / \hbar$ has been replaced by $\omega_{0}$, cf. Eq. (26) of Ref. 3(b).

The Hall conductivity $\sigma_{y x}(0) \equiv \sigma_{y x}=-\sigma_{x y}$ is readily evaluated using the expressions for the velocity matrix elements between the states (11) together with those for $V_{n, n \pm 1}$ as given by Eq. (12) and the matrix elements between the unperturbed states. Neglecting terms of order $B_{0}^{2}$ in the product $\left\langle\zeta\left|v_{y}\right| \zeta^{\prime}\right\rangle\left\langle\zeta^{\prime}\left|v_{x}\right| \zeta\right\rangle$ the result is

$\sigma_{y x}=\frac{e^{2}}{h} \frac{2 \ell^{2}}{a} \sum_{n}(n+1) \int_{0}^{a / \ell^{2}} d k_{y} \frac{f_{n, k_{y}}-f_{n+1, k_{y}}}{\left[1+\lambda_{n}^{\prime} \cos \left(K x_{0}\right)\right]^{2}}$

where $\lambda_{n}^{\prime}=\left(\omega_{0} / \omega_{c}\right) \exp (-u / 2)\left[G_{n+1}(u)-G_{n}(u)\right]$. The correspondence with the electric case, ${ }^{3}$ which involves $\lambda_{n}=\left(V_{0} / \hbar \omega_{c}\right) e^{-u / 2} L_{n+1}^{-1}$ rather than $\lambda_{n}^{\prime}$, is that for equal modulation strengths, i.e., for $V_{0}=\hbar \omega_{0}$, we have

$$
\lambda_{n}^{\prime}=\lambda_{n} / 2+\left(\omega_{0} / \omega_{c}\right) e^{-u / 2}\left[L_{n}^{1}(u)-L_{n-1}^{1}(u)\right] .
$$

The main difference of Eq. (16) from the corresponding result of Ref. 5, Eq. (18), is the absence of corrections, in the numerator, linear in $B_{0}$. We have verified that these corrections vanish identically as they are proportional to the difference $V_{n m}-V_{n m}^{*}$ and $V_{n m}=\langle n|V| m\rangle$ is a real quantity for the perturbation $V=\left(\omega_{0} / K\right)\left(p_{y}+e B x\right) \sin (K x)$. The same holds for the electric modulation. Consequently the result of Ref. 5 is incorrect.

The resistivity tensor $\rho=\sigma^{-1}$ is evaluated from the conductivity tensor using the standard expressions $\rho_{x x}=\sigma_{y y} / S, \rho_{y y}=\sigma_{x x} / S$, and $\rho_{x y}=-\sigma_{y x} / S$ with $S=\sigma_{y y} \sigma_{x x}-\sigma_{x y} \sigma_{y x}$.

\section{B. Asymptotic expressions}

For $n \gg 1$, i.e., when many Landau levels are occupied, the results of Sec. III A can be cast into a form that exhibits explicitly both the Weiss (at low $B$ ) and the Shubnikov-de Haas (at higher $B$ ) oscillations. The procedure has been detailed in Ref. 3(b) and consists in using the asymptotic expressions for the Laguerre polynomials, for $n \gg 1$, and of the density of states $D(E)=D_{0}\left[1-2 \exp \left(-\pi / \omega_{c} \tau_{f}\right) \cos \left(2 \pi E / \hbar \omega_{c}\right)\right]$ with the prescription $\sum_{n} \rightarrow 2 \pi \ell^{2} \int D(E) d E$. The parameter $\tau_{f}$ is the electron quantum lifetime. Following verbatim this procedure and retaining only the leading terms we obtain that Eq. (10) takes the form

$$
\frac{\sigma_{y y}^{\mathrm{dif}}}{\sigma_{0}}=\frac{a k_{F}}{2 \pi^{2}} \frac{\hbar \omega_{0}}{\hbar \omega_{c}} \frac{\hbar \omega_{0}}{E_{F}}\left[G-2 e^{-\pi / \omega_{c} \tau_{f}} A\left(T / T_{c}\right) \cos \left(\frac{2 \pi E_{F}}{\hbar \omega_{c}}\right) \sin ^{2}\left(\frac{2 \pi R_{c}}{a}-\frac{\pi}{4}\right)\right],
$$

where

$$
G=\left[1-A\left(T / T_{a}\right)\right] / 2+A\left(T / T_{a}\right) \sin ^{2}\left(2 \pi R_{c} / a-\pi / 4\right) .
$$

Here $\sigma_{0}=n_{e} e^{2} \tau / m^{*}$ is the conductivity at zero magnetic field $B$ and $A(x)=x / \sinh (x)$. The characteristic temperatures $T_{a}$, for the Weiss oscillations, and $T_{c}$, for the SdH oscillations, are defined by $k_{B} T_{a}=\left(\hbar \omega_{c} / 4 \pi^{2}\right) a k_{F}$ and $k_{B} T_{c}=\hbar \omega_{c} / 2 \pi^{2}$, respectively, with $k_{F}$ being the Fermi wave vector. In typical experiments we have $T_{a} / T_{c}=a k_{F} / 2 \gg 1$; e.g., for $n_{e}=3 \times 10^{11} / \mathrm{cm}^{2}$ and $a=3500 \AA$, we have $a k_{F} \approx 24$. As a result the $\mathrm{SdH}$ oscillations disappear much faster with temperature than the Weiss oscillations. We further remark that in comparison with the electric case, cf. Eq. (34) of Ref. 3(b), the present result differs essentially by a term $\left(a k_{F} / 2 \pi\right)^{2} \approx 60$ in the prefactor. Correspondingly the amplitude will be larger in the magnetic case by this factor.

The asymptotic expression for the collisional conductivity can be obtained along the same lines. The result is 


$$
\frac{\sigma_{x x}^{\mathrm{col}}}{\sigma_{0}} \approx \frac{1}{\omega_{c}^{2} \tau^{2}}\left\{1+\frac{3}{\sqrt{2} \pi} \frac{\omega_{0}^{2}}{\omega_{c}^{2}} \sqrt{\frac{E_{F}}{\hbar \omega_{c}}}\left[F-2 e^{-\pi / \omega_{c} \tau_{f}} A\left(T / T_{c}\right) \cos \left(\frac{2 \pi E_{F}}{\hbar \omega_{c}}\right) \cos ^{2}\left(\frac{2 \pi R_{c}}{a}-\frac{\pi}{4}\right)\right]\right\}
$$

where $F$ is given by Eq. (19) after replacing $\sin ^{2}()$ by $\cos ^{2}()$. Equation (20) has the same structure as Eq. (37) of Ref. 3(b) that pertains to an electric modulation.

\section{Numerical results}

A detailed numerical evaluation of the resistivity tensor, using Eqs. (10), (13), and (16) for the component of the conductivity tensor, can proceed as in Ref. 3(b). However, as explained above the results of the magnetic modulation scale with those of the electric one or have the same structure. Therefore, the relevant calculation will not be repeated. Moreover, as indicated by the good agreement between the exact numerical and the classical result for the bandwidth, cf. Fig. 1, a detailed numerical evaluation will change the asymptotic results presented below very little. In addition, It has been found ${ }^{3(b)}$ that the collisional contribution to the conductivity is about ten times smaller than the diffusive one. With that in mind we will give the results for the correction to the resistivity $\Delta \rho_{x x} / \rho_{0} \equiv \Delta \rho$, due to the modulation including only the diffusion contribution, i.e., Eq. (18). The quantity $\rho_{0}$ is the resistivity at zero magnetic field. The other component $\rho_{y y}$ only has contributions from the collisional term and as such the Weiss oscillations are much weaker than in $\rho_{x x}$. The same holds for $\rho_{y x}$. Therefore we will concentrate on the numerical results for $\rho_{x x}$.

In Fig. 2 we plot $\Delta \rho$ as a function of the magnetic field

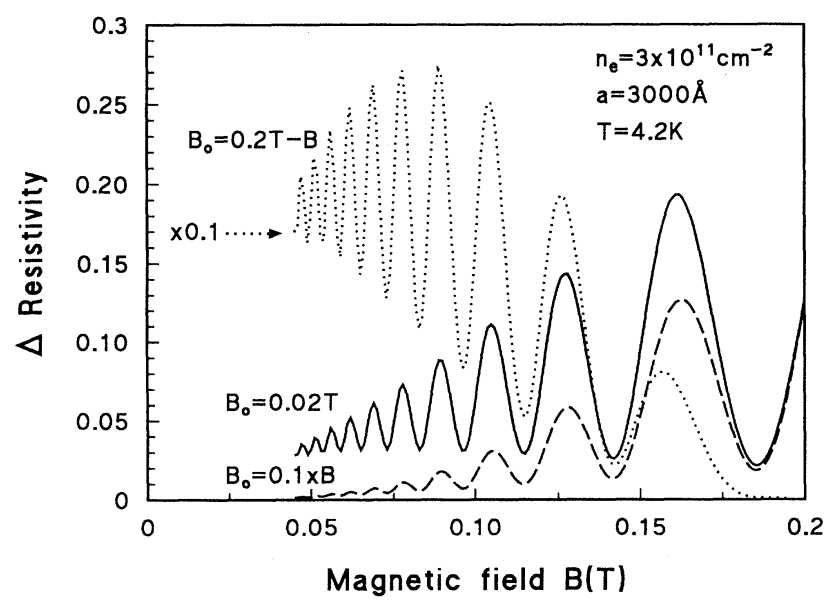

FIG. 2. The correction $\Delta \rho_{x x} / \rho_{0} \equiv \Delta \rho$ to the resistivity $\rho_{x x}$ due to the modulation, scaled by the $B=0$ resistivity $\rho_{0}$, as function of the magnetic field. The solid curve corresponds to a constant $B_{0}=0.02 \mathrm{~T}$, the dotted one (scaled down by a factor of 10) to $B_{0}=0.2 \mathrm{~T}-B$, and the dashed curve to $B_{0}=0.1 B$. The electron density is $n_{e}=3 \times 10^{11} \mathrm{~cm}^{-2}$, the modulation period $a=3000 \AA$, and the temperature $T=4.2$ K.
$B$ for values of $B$ such that only the Weiss oscillations appear. The solid curve is for constant $B_{0}=0.02 \mathrm{~T}$, the dashed curve for $B_{0}=0.1 B$, and the dotted one (scaled down by a factor of 10 ) for $B_{0}=0.2 \mathrm{~T}-B$. The latter is expected to occur in the case of type-I superconducting strips with a critical field of about $0.2 \mathrm{~T}$. The temperature is $T=4.2 \mathrm{~K}$, the electron density $n_{e}=3 \times 10^{11} / \mathrm{cm}^{2}$, and the period of the modulation $a=3000 \AA$. As can be seen (1) the position of the extrema depends very little on the assumed form of $B_{0},(2)$ there is no qualitative difference between the solid and dashed curves since in either case the inequality $B_{0} \ll B$ guarantees the validity of the perturbation treatment, and (3) the amplitude of the dashed curve is about a factor of 3 smaller than that of the solid one.

In Fig. 3 we plot $\Delta \rho$ as a function of $B$ for constant $B_{0}=0.02 \mathrm{~T}$ for three different temperatures. The other (indicated) parameters are the same as those in Fig. 2. At very low $B(B \leq 0.5 \mathrm{~T})$ we have the Weiss oscillations whereas at higher $B$ the $\mathrm{SdH}$ ones appear clearly. We see clearly that the latter disappear fast with the temperature whereas the former remain practically temperature independent up to $T=10 \mathrm{~K}$. This is related to the critical temperatures $T_{a}$ and $T_{c}$ introduced earlier and has been discussed extensively in Ref. 3(b) for electric modulations.

\section{ELECTRIC AND MAGNETIC MODULATIONS}

Both of the possible methods of magnetic modulation mentioned in the Introduction are expected to be accompanied by an electric one since gates are applied to the

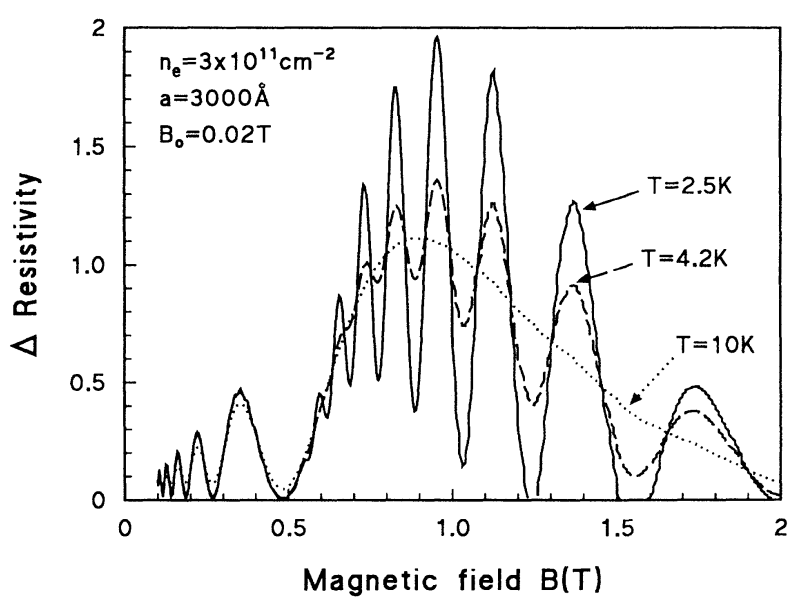

FIG. 3. $\Delta \rho$ as a function of the magnetic field for constant $B_{0}=0.02 \mathrm{~T}$ and three different temperatures as indicated. The other parameters are the same as those of Fig. 2. 
heterostructure. We are thus led to study how the results of Sec. III are modified when an electric modulation with the same period is present. Two cases are of interest: that where the two modulations are in phase and that where they are out of phase.

\section{A. In-phase modulations}

If a weak electric modulation described by the periodic potential $V_{0} \cos (K x)$, which is in phase with the magnetic one, is present Eq. (2) will have an additional term $V_{0} F_{n}(u) \cos \left(K x_{0}\right)$. As a result $v_{y}$, given by Eq. (6), will have an additional term $-\left(2 V_{0} / \hbar K\right) u F_{n}(u) \sin \left(K x_{0}\right)$. The bandwidth will be the sum of the two bandwidths. At the Fermi energy the latter is given by the sum of those of Eqs. (4a) and (4b) and is equal to

$$
2 V_{0} \sqrt{2 / \pi K R_{c}} \sqrt{1+\delta^{-2}}\left|\sin \left(2 \pi R_{c} / a-\pi / 4+\phi\right)\right|
$$

where $\delta=2 \pi V_{0} / a k_{F} \hbar \omega_{0}=\tan (\phi)$. Notice that the flatband condition now reads $2 R_{c} / a=i+1 / 4-\phi / \pi$ and depends on the relative strength of the two modulations.

The changes mentioned above will be reflected in the transport coefficients as well. As an example the diffusion contribution to the conductivity corresponding to Eq. (10) now takes the form

$$
\begin{aligned}
\sigma_{y y}^{\mathrm{dif}} \approx \frac{e^{2}}{h} \frac{2 \pi^{2} \tau}{\hbar} \frac{\ell^{2}}{a^{2}} \sum_{n}[ & \left.\hbar \omega_{0} G_{n}(u)+V_{0} F_{n}(u)\right]^{2} \\
& \times\left(-\frac{\partial f(E)}{\partial E}\right)_{E=E_{n}}
\end{aligned}
$$

and its asymptotic expression, obtained in the manner described earlier, reads

$$
\begin{aligned}
\frac{\sigma_{y y}^{\text {dif }}}{\sigma_{0}} \approx \frac{a k_{F}}{2 \pi^{2}} \frac{\hbar \omega_{0}}{\hbar \omega_{c}} \frac{\hbar \omega_{0}}{E_{F}}\left(1+\delta^{2}\right)\left\{1-A\left(T / T_{a}\right)+\right. & {\left[A\left(T / T_{a}\right)-2 e^{-\pi / \omega_{c} \tau_{f}} A\left(T / T_{c}\right) \cos \left(\frac{2 \pi E_{F}}{\hbar \omega_{c}}\right)\right] } \\
& \left.\times \sin ^{2}\left(\frac{2 \pi R_{c}}{a}-\frac{\pi}{4}+\phi\right)\right\} .
\end{aligned}
$$

As can be seen the influence of the combined effect of the two modulations is to introduce the phase factor $\phi$ in the Weiss oscillations.

In Fig. 4 we plot $\Delta \rho$ as a function of $B$ (solid curve) for $B_{0}=0.02 \mathrm{~T}$ and $V_{0}=0.2 \mathrm{meV}$. The other parameters are the same with those of Fig. 2. For comparison we

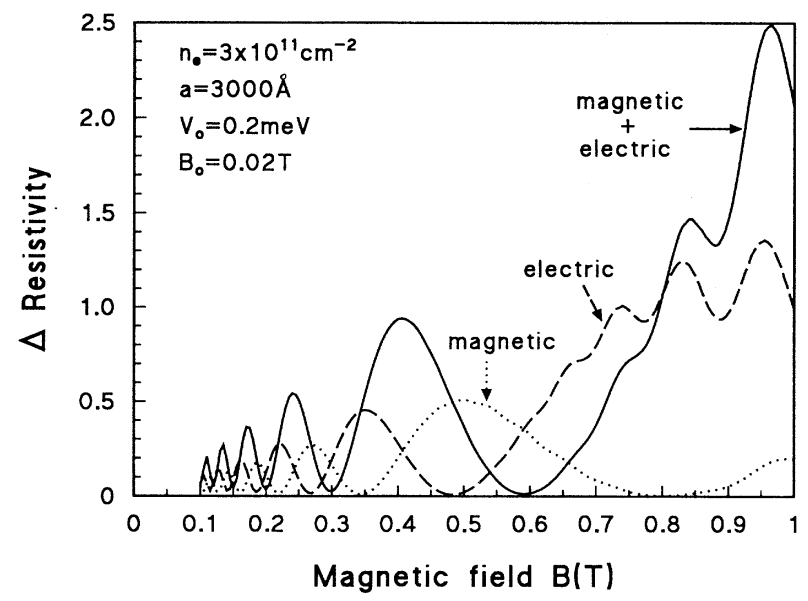

FIG. 4. $\Delta \rho$ as a function of the magnetic field (solid curve) when a magnetic and an electric in-phase modulation are present. The modulation strengths are $B_{0}=0.02 \mathrm{~T}$ and $V_{0}=0.2 \mathrm{meV}$. The other parameters are the same as in Fig. 2 . The dotted and dashed curves show $\Delta \rho$ for the same magnetic and electric modulation alone, respectively. show the results when only the magnetic (dotted curve) or the electric (dashed curve) modulation is present. In line with Eq. (23) that was used for the evaluation of $\Delta \rho$ the solid curve is shifted from the other curves due to the phase factor $\phi$. We also remark about the $\pi / 2$ phase difference between the dashed and dotted curves which

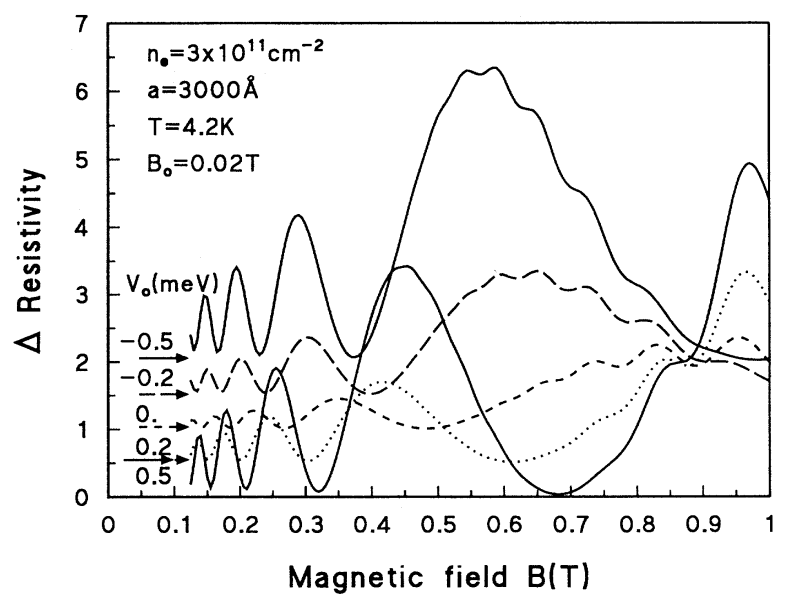

FIG. 5. $\Delta \rho$ as a function of the magnetic field when a magnetic and an electric in-phase modulation are present. $B_{0}=0.02 \mathrm{~T}$ is kept constant while $V_{0}$ varies between negative and positive values. The value of $V_{0}$ is indicated at the begining of each curve that is shifted upwards from the previous one by 0.5 as indicated by the arrows. 
reflects that of the corresponding bandwidths shown in Fig. 1.

The dependence of $\Delta \rho$ on $B$ and the phase factor $\phi$ is shown in Fig. 5. $B_{0}$ is again kept constant $\left(B_{0}=0.02 \mathrm{~T}\right)$ but $V_{0}$ is varied as indicated between positive and negative values; $\delta$ and $\phi$ change accordingly. We notice that (1) the position of the peaks depends on the specific value of $\delta$ used and (2) there is a $\pi$ phase difference between large positive and large negative values of $\delta$.

\section{B. Out-of-phase modulations}

If light is shined on top of a magnetically modulated heterostructure the light pulses will ionize $D X$ centers in the $\mathrm{Al}_{x} \mathrm{Ga}_{1-x} \mathrm{As}$ layer between the gates (e.g., strips of magnetic materials) where $B_{0}=0$. This will create an electric modulation $\pi / 2$ out of phase with the magnetic one. Assuming that the electric modulation of the gates is much smaller than that of the light, something that can be achieved by contacting the gates, we are led to consider transport in the presence of the two modulations that are $\pi / 2$ out of phase.

We take the same electric modulation $V_{0} \cos (K x)$ and assume a magnetic one given by $B(x)=B+B_{0} \sin (K x)$. To first order in $V_{0}$ and $B_{0}$ the eigenvalue $E_{n k_{y}}$ and velocity $v_{y}$ are given by

$$
\begin{aligned}
E_{n k_{y}}= & (n+1 / 2) \hbar \omega_{c}+\hbar \omega_{0} G_{n}(u) \sin \left(K x_{0}\right) \\
& +V_{0} F_{n}(u) \cos \left(K x_{0}\right)
\end{aligned}
$$

and

$$
\begin{array}{r}
v_{y}=-(2 u / \hbar K)\left[\hbar \omega_{0} G_{n}(u) \cos \left(K x_{0}\right)\right. \\
\left.-V_{0} F_{n}(u) \sin \left(K x_{0}\right)\right],
\end{array}
$$

respectively. These apparently smooth changes can have important consequences for transport. For instance, the combined bandwidth at the Fermi energy now is equal to

$2 V_{0} \sqrt{2 / \pi K R_{c}} \sqrt{1+\left(\delta^{-2}-1\right) \sin ^{2}\left(2 \pi R_{c} / a-\pi / 4\right)}$.

If $\delta= \pm 1$ the bandwidth no longer oscillates as a function of the magnetic field, i.e., the Weiss oscillations are washed out.

The result for the diffusion contribution reads

$$
\begin{aligned}
& \sigma_{y y}^{\mathrm{dif}}=\frac{e^{2}}{h} \frac{2 \pi^{2} \tau}{\hbar} \frac{\ell^{2}}{a^{2}} \sum_{n}\left\{\left[V_{0} F_{n}(u)\right]^{2}+\left[\hbar \omega_{0} G_{n}(u)\right]^{2}\right\} \\
& \times\left(-\frac{\partial f(E)}{\partial E}\right)_{E=E_{n}}
\end{aligned}
$$

There is no cross term involving the product $\hbar \omega_{0} V_{0}$ in Eq. (27), as expected from Eqs. (7) and (25), because the integral over $k_{y}$ vanishes for this term if we neglect the very weak $k_{y}$ dependence of the argument of the Fermi functions.

The asymptotic expression for $\sigma_{y y}^{\text {dif }}$ is

$$
\frac{\sigma_{y y}^{\mathrm{dif}}}{\sigma_{0}}=\frac{\hbar \omega_{0}}{E_{F}} \frac{\hbar \omega_{0}}{\hbar \omega_{c}} \frac{a k_{F}}{2 \pi^{2}}\left\{G+\delta^{2} F-2 e^{-\pi / \omega_{c} \tau_{f}} A\left(T / T_{c}\right) \cos \left(\frac{2 \pi E_{F}}{\hbar \omega_{c}}\right) D\right\}
$$

where

$$
D=\delta^{2}-\left(\delta^{2}-1\right) \sin ^{2}\left(\frac{2 \pi R_{c}}{a}-\frac{\pi}{4}\right)
$$

Comparing Eq. (29) with Eq. (23) we see that changing $\delta$ does not change the position of the extrema of $\Delta \rho$ as a function of $B$ since the phase factor $\phi$ is absent from Eq. (29). This is illustrated in Fig. 6 where $\Delta \rho$ is plotted as a function of $B$ for $B_{0}=0.02 \mathrm{~T}$ with variable $V_{0}$. For the upper two curves we have $\delta>1$ whereas for the lower two curves $\delta$ is smaller than 1 . This results in an antiphase between the two groups of curves and reflects the corresponding behavior of the bandwidth as given by Eq. (26).

\section{CONCLUSIONS}

We calculated the magnetoconductivity tensor for a 2DEG in the presence of a weak modulation of the magnetic field. The magnetoresistivity tensor exhibits Weiss

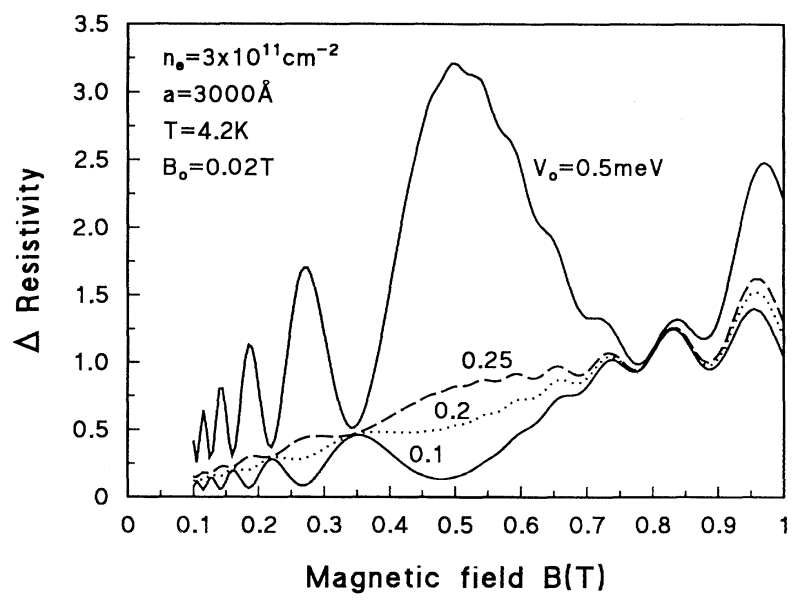

FIG. 6. $\Delta \rho$ as a function of the magnetic field when a magnetic and an electric, $\pi / 2$ out-of-phase modulation, are present. The values of $V_{0}$ in $\mathrm{meV}$ are indicated on each curve and the other parameters are the same as in Fig. 5. 
oscillations which are different from those from an electric modulation: (1) there is a $\pi / 2$ phase shift between the oscillations and (2) for equal modulation strengths (i.e., $V_{o}=\hbar \omega_{c}$ ) the oscillation amplitude in the magnetic case is much larger than for the electric case.

In a real experimental system we expect that an electric modulation will inherently be present with each magnetic modulation. Therefore we have also studied the case in which both types of modulations are present in a 2DEG. We found that if both modulations are in phase the extremal positions of the Weiss oscillations are shifted continuously with increasing strength of the electric oscillation. On the other hand, when the electric and magnetic modulation are $\pi / 2$ out of phase a different behavior is found in which with increasing electric modulation strength the position of the Weiss oscillations are not influenced but the amplitude is. If $B_{0}$ is kept constant for a critical value of the strength of the electric modulation the Weiss oscillations disappear and with a further increase of the modulation strength the Weiss oscillations reappear but now the maxima appear at the position of the previous minima.

\section{ACKNOWLEDGMENTS}

This work was supported by NSERC Grant No. ICR7R 106 and by the Collaborative Research Grant: NATO: $5-2-05 /$ RG No. 890123. One of us (F.M.P.) is supported by the Belgian National Science Foundation.
${ }^{1}$ D. Weiss, K. von Klitzing, K. Ploog, and G. Weiman, Europhys. Lett. 8, 179 (1989); R.R. Gerhardts, D. Weiss, and K. von Klitzing, Phys. Rev. Lett. 62, 1173 (1989); R.W. Winkler, J.P. Kotthaus, and K. Ploog, ibid. 62, 1177 (1989); E.S. Alves et al., J. Phys. Condens. Matter 1, 8257 (1989); P.H. Beton et al., Phys. Rev. B 42, 9229 (1990); 42, 9689 (1990); H. Fang and P.J. Stiles, ibid. 41, 10171 (1990); A. Toriumi, K. Ismail, M. Burkhardt, D. A. Antoniadis, and H. I. Smith, ibid. 41, 12346 (1990).

${ }^{2}$ C.T. Cui, V. Fessatidis, and N.J.M. Horing, Phys. Rev. Lett. 63, 2598 (1989); P. Středa and A.H. MacDonald, Phys. Rev. B 41, 11892 (1990); C.W.J. Beenakker, Phys. Rev. Lett.
62, 2020 (1989); C. Zhang and R.R. Gerhardts, Phys. Rev. B 41, 12850 (1990).

${ }^{3}$ (a) P. Vasilopoulos and F.M. Peeters, Phys. Rev. Lett. 63, 2120 (1989); (b) F.M. Peeters and P. Vasilopoulos, Phys. Rev. B 46, 4667 (1992); (c) ibid. 42, 5899 (1990).

${ }^{4}$ P. Vasilopoulos and F.M. Peeters, Superlatt. Microstruct. 7, 393 (1990).

${ }^{5}$ D. P. Xue and G. Xiao, Phys. Rev. B 45, 5986 (1992).

${ }^{6}$ X. G. Wu and S. Ulloa, Solid State Commun. 82, 945 (1992).

${ }^{7}$ A. K. Heym et al., Pis'ma Zh. Eksp. Teor. Fiz. 51, 107 (1990) [JETP Lett. (1990)]. 